IFT-UAM/CSIC-97-3

hep-th/9711074

\title{
Elliptic Singularities, $\theta$-Puzzle and Domain Walls
}

\author{
César Gómez \\ Instituto de Matemáticas y Física Fundamental, CSIC, \\ Serrano 123, 28006 Madrid, Spain \\ and \\ Instituto de Física Teórica (UAM-CSIC), C-XVI, Universidad Autónoma de Madrid, \\ Cantoblanco 28049, Madrid, Spain
}

\begin{abstract}
We study $N=1$ four dimensional gluodynamics in the context of M-theory compactifications on elliptically fibered Calabi-Yau fourfolds. Gaugino condensates, $\theta$-dependence, Witten index and domain walls are considered for singularities of type $\hat{A}_{n-1}$ and $\hat{D}_{n+4}$. It is shown how the topology of intersections among the irreducible components defining the singular elliptic fiber, determine the entanglement of vacua and the appareance of domain walls.
\end{abstract}




\section{Introduction and Summary}

$N=1$ four dimensional gauge theories can be obtained by means of $F$-theory compactifications [1, 2, 3, 国 on elliptically fibered Calabi Yau fourfolds $X$ :

$$
E \rightarrow X \rightarrow B
$$

or equivalently by $M$-theory compactifications on $X$ in the limit $\operatorname{Vol}(E)=0$. Assuming Kodaira classification [5] of singular fibers extends to the case of elliptically fibered CalabiYau fourfolds and working locally, in the spirit of geometric engineering [6, 7], we can get $N=1$ four dimensional gauge theories with $A D E$ type of gauge groups. In this paper we will consider, from that point of view, some problems concerning $N=1$ super gluodynamics, in particular gaugino condensates, the $\theta$-puzzle [8], the Witten index [9] and domain walls [10, 11, 12]. These issues has been recently considered, in the context of intersecting branes in reference 14].

Chiral symmetry breaking in $N=1$ pure gluodynamics, i. e., non vanishing condensates $\langle\lambda \lambda>$ for gluino bilinears, has been derived long time ago using different methods. In $S U(n)$ pure super Yang-Mills, instantons contribute to condensates $<\lambda \lambda\left(x_{1}\right) \ldots \lambda \lambda\left(x_{n}\right)>$ and they are, after integration over the size of the instanton, independent of the positions $x_{1}, x_{2}, \ldots, x_{n}$ [15, 16, 17]. Through cluster decomposition we can get a set of values for $\langle\lambda \lambda\rangle$,

$$
<\lambda \lambda>_{j}=C \Lambda^{3} e^{2 \pi i j / n} e^{i \theta / n}
$$

with $j=0, \ldots, n-1, C$ some constant, $\Lambda$ the $S U(n) N=1$ scale and $\theta$ the $\theta$-vacua parameter. A similar result [18] can be directly derived, for $S U(n)$ gauge groups, using 't Hooft's torons [19]. In both cases this way of computing the condensates is doubtful. Namely, using cluster, we must implicetely consider the contribution of instantons of arbitrarily large size, which is certainly beyond the regime where semiclassical analysis is reliable. The same happens in the toron computation, where an infinite volume limit must be performed before reaching the result (2). A different approach to the computation of $\langle\lambda \lambda\rangle$ is in the weak coupling regime [20] where the holomorphy dependence on an auxiliary mass is used. These two procedures do not coincide on the value of the 
constant in (2). Recently a possible way out to this puzzle has been proppossed in reference [13] where the existence of an extra vacua without chiral symmetry breaking is suggested. Leaving for a while these issues the result (2) contains already some interesting peculiarities. If the different values for $\langle\lambda \lambda\rangle_{i}$ are identified with different vacua, the change $\theta \rightarrow \theta+2 \pi$ transforms the vacuum $i$ into the vacuum $i+1$. Moreover the number of $\langle\lambda \lambda\rangle$ values coincides, for $S U(n)$ with the Witten index $\operatorname{tr}(-1)^{F}$, but this is not the case for $O(N)$ groups where the number of $\langle\lambda \lambda\rangle$ values is $N-2$ while the Witten index is $\left[\frac{N}{2}\right]+1$.

Since non vanishing values for $\langle\lambda \lambda\rangle$ are connected with a non trivial superpotential for $N=1$ gluodynamics [21], and taking into account that vertical instantons [1] in M-theory survive in the $N=1$ four dimensional limit, it is natural looking for a superpotential that agrees with (2), directly in M-theory. A first step in this direction was already taken in reference [7]. In this approach the geometry of Kodaira singularities is crucial. For an elliptically fibered Calabi-Yau fourfold, M-theory instantons [1] are defined as divisors, i. e., six cycles, with holomorphic Euler characteristic $\chi=1$. If the instanton is vertical, then we can safely use it as a contribution to the four dimensional superpotential. In the Kodaira classification [5], the singular fibers are defined by 2-cycles $\mathcal{C}$ of the type:

$$
\mathcal{C}=\sum_{i} n_{i} \Theta_{i}
$$

with $\Theta_{i}^{2}=-2$, non singular rational curves, $n_{i}$ integer numbers and the intersection matrix $\Lambda_{i j}=\left(\Theta_{i} \cdot \Theta_{j}\right)$ one to one related to some affine Dynkin diagram. Moreover, the self intersection $\left(\mathcal{C}^{2}\right)$ is equal to zero, which is characteristic of elliptic singularities. In a Calabi-Yau fourfold $X$ the singular locus will be a four dimensional manifold $C$ and we will assume that the singular fiber is trivially fibered over $C$. In addition, we will think of $C$ as an Enriques surface. With these assumptions, it is easy to get M-theory instantons $D_{i}$ associated with the $\Theta_{i}$ in (3) [7] all of them with $\chi=1$, and therefore with two fermionic zero modes. Following [1] we associate with each of these divisors a scalar field $\phi_{D_{i}}$ transforming under chiral $U(1)$ as:

$$
\phi_{D_{i}} \rightarrow \phi_{D_{i}}+\alpha
$$


The topological sum defined as $\sum n_{i} \phi_{D_{i}}$ transforms as $\sum n_{i} \phi_{D_{i}} \rightarrow \sum n_{i} \phi_{D_{i}}+$ Cox $\alpha$, where Cox is the Coxeter number of the diagram defined as the total number of irreducible components. This is the transformation rule of the $\theta$ parameter in $N=1$ super gluodynamics as derived from the $U(1)$ axial anomaly. Thus we define the $\theta$ parameter as the topological sum $\sum n_{i} \phi_{D_{i}} \bmod (2 \pi)$. For the $S U(n)$ case corresponding to $\hat{A}_{n-1}$ singularities, we have under $\mathbf{Z}_{n}$ transformations, $\sum_{i=0}^{n-1} \phi_{D_{i}} \rightarrow \sum_{i=0}^{n-1} \phi_{D_{i}} \bmod (2 \pi)$, which can be solved by $\phi_{D_{j}}=\frac{2 \pi j}{n}+\frac{\theta}{n}$, with $\theta \in[0,2 \pi]$ undetermined. This corresponds to the geometry of $\hat{A}_{n-1}$ Kodaira singularities, where under chiral $\mathbf{Z}_{n}$ the ireducible components transform cyclically as $\Theta_{i} \rightarrow \Theta_{i+1}$.

By standard M-theory instanton computations [1], we can associate with each divisor $D_{j}$, such that $\chi\left(D_{j}\right)=1$ a gaugino condensate $<\lambda \lambda>_{\theta}^{j}$. From (幽), and the definition of $\theta$ as a topological sum, we easily get $<\lambda \lambda>_{\theta}^{j}=\left\langle\lambda^{\prime} \lambda^{\prime}>_{\theta+\alpha n}^{j}\right.$, for $\lambda^{\prime}=e^{i \alpha / 2} \lambda$ (notice that in four dimensions $\theta$ play a similar role to the three dimensional Goldstone boson 22] which in M-theory on Calabi-Yau fourfolds can be identified with the scalar field $\left.\phi_{D_{i}}\right)$. In summary, we start with divisors $D_{j}$, with $\chi\left(D_{j}\right)=1$ in an elliptically fibered Calabi-Yau fourfold. By purely geometrical arguments [1], we derive the transformation rule (4). Next, we consider a singular fiber of Kodaira type $A D E$ as defined by a cycle of the type (3). Then the topological sum $\sum n_{i} \phi_{D_{i}}$ reproduces the chiral transformations of $\theta$ dictated by the $U(1)$ axial anomaly of $A D E N=1$ four dimensional gauge theories. At this point, the reader can wonder where four dimensions enter the argument. In the three dimensional limit defined by $\operatorname{Vol}(E)=\infty$ the singularity, as we will discuss later, becomes rational, of type $A_{n-1}$, with $n-1$ irreducible components, and the topological sum does not possess any clear physical meaning.

The $\theta$-puzzle appears as a consequence of the following fact. If the $\theta$ angle (we reduce here the discussion to $S U(n))$ is defined as the topological sum $\sum_{i=0}^{n-1} \phi_{D_{i}}$, then it is tempting to think of $\theta$ as the scalar field $\phi_{\mathcal{D}}$, associated to the six cycle $\mathcal{D}$, defined by trivially fibering the $\hat{A}_{n-1}$ cycle $\mathcal{C}=\sum_{i=0}^{n-1} \Theta_{i}$ over the singular locus $C$. Then the transformation rule under chiral $U(1), \theta \rightarrow \theta+n \alpha$ will mean that $\chi(\mathcal{D})=n$. This is the mathematical way to translate the physical argument relating $\theta$ to instanton tunneling and instantons to condensates of the type $<\lambda \lambda\left(x_{1}\right) \ldots \lambda \lambda\left(x_{n}\right)>$. Where is the loophole of 
this way of thinking?. The loophole is mathematically very clear, namely the holomorphic Euler characteristic of the 6 -cycle $\mathcal{D}$ is zero, and not $n$, and therefore $\theta$ is not the field $\phi \mathcal{D}$. The intuitive way to understand it, is simply observing that $\mathcal{C}$ is not just the union of $n$ disconnected components $\Theta_{i}$, but a very specific set of intersections that, by the Grothendieck-Riemann-Roch theorem [23], leads to $\chi(\mathcal{D})=0$.

What are the consequences of $\chi(\mathcal{D})=0$ from a physical point of view?. Intuitively we can think of $\chi(\mathcal{D})=0$ as reflecting a complete soaking up of the fermionic zero modes associated to the components $\Theta_{i}$. This approach allows us to associate to each intersection $\left(\Theta_{i} \cdot \Theta_{i+1}\right)$ a term soaking up two fermionic zero modes, of the same order of magnitude as the $\langle\lambda \lambda\rangle$ condensate. These intersection terms are perfect candidates for domain walls whose existence is the physical way to solve the $\theta$-puzzle. In other words, $\chi(\mathcal{D})=0$ implies the entanglement of the different vacua, entanglement that is physically mediated by the intersection terms. The extension of this entanglemnet mechanism to $N=0$ supersymmetry would be extremely interesting for explaining the $\theta$ dependence in the Witten-Veneziano formula 24, 25].

Thinking in terms of branes in M-theory, instantons are interpreted [1] as fivebranes wrapped on six cycles $D_{j}$; thus, the intersection term should be interpreted as a five brane wrapped on $C \times\left(\Theta_{i} \cdot \Theta_{i+1}\right)$, which produces, in four dimensions, a wall of the appropiate tension, interpolating the $i$ and $i+1$ vacua. Moreover, the two fermionic zero modes associated to the intersection term seems to be the right BPS signal of the domain wall. The extension of the previous arguments to $O(N)$ gauge groups, i. e., to $\hat{D}$ type singularities, will present some extra difficulties that we will discuss.

In summary the solution to the $\theta$-puzzle and the existence of domain walls are related to the topology of intersections of the irreducible components defining singular elliptic fibers.

\section{Local Models for Elliptic Fibrations.}

Let $V$ be an elliptic fibration,

$$
\Phi: V \rightarrow \Delta
$$


with $\Delta$ an algebraic curve, and $\Phi^{-1}(a)$, with $a$ any point in $\Delta$, an elliptic curve. Let us denote $\left\{a_{\rho}\right\}$ the finite set of points in $\Delta$ such that $\Phi^{-1}\left(a_{\rho}\right)=\mathcal{C}_{\rho}$ is a singular fiber. Each singular fiber $\mathcal{C}_{\rho}$ can be written as

$$
\mathcal{C}_{\rho}=\sum_{i} n_{i \rho} \Theta_{i \rho}
$$

where $\Theta_{i \rho}$ are non singular rational curves, with $\Theta_{i \rho}^{2}=-2$, and $n_{i \rho}$ are integer numbers. Different types of singularities are characterized by (6) and the intersection matrix $\left(\Theta_{i \rho} . \Theta_{j \rho}\right)$. All different types of Kodaira singularities satisfy the relation

$$
\mathcal{C}_{\rho}^{2}=0
$$

Let $\tau(u)$ be the elliptic modulus of the elliptic fiber at the point $u \in \Delta$. For each path $\alpha$ in $\Pi_{1}\left(\Delta^{\prime}\right)$, with $\Delta^{\prime}=\Delta-\left\{a_{\rho}\right\}$, we can define a monodromy transformation $S_{\alpha}$, in $\operatorname{Sl}(2, \mathbf{Z})$, acting on $\tau(u)$ as follows:

$$
S_{\alpha} \tau(u)=\frac{a_{\alpha} \tau(u)+b_{\alpha}}{c_{\alpha} \tau(u)+d_{\alpha}} .
$$

Each type of Kodaira singularity is characterized by a particular monodromy matrix.

In order to define an elliptic fibration [5], the starting point will be an algebraic curve $\Delta$, that we will take, for simplicity, to be of genus zero, and a meromorphic function $\mathcal{J}(u)$ on $\Delta$. Let us assume $\mathcal{J}(u) \neq 0,1, \infty$ on $\Delta^{\prime}=\Delta-\left\{a_{\rho}\right\}$. Then, there exists multivalued holomorphic function $\tau(u)$, with $\operatorname{Im} \tau(u)>0$, satisfying $\mathcal{J}(u)=j(\tau(u)$ ), with $j$ the elliptic modular $j$-function on the upper half plane. As above, for each $\alpha \in \Pi_{1}\left(\Delta^{\prime}\right)$ we define a monodromy matrix $S_{\alpha}$, acting on $\tau(u)$ in the form defined by (8). Associated to these data we will define an elliptic fibration, (5). In order to do that, let us first define the universal covering $\tilde{\Delta}^{\prime}$, of $\Delta^{\prime}$, and let us identify the covering transformations of $\tilde{\Delta}^{\prime}$ over $\Delta^{\prime}$, with the elements in $\Pi_{1}\left(\Delta^{\prime}\right)$. Denoting by $\tilde{u}$ a point in $\tilde{\Delta}^{\prime}$, we define, for each $\alpha \in \Pi_{1}\left(\Delta^{\prime}\right)$, the covering transformation $\tilde{u} \rightarrow \alpha \tilde{u}$, by

$$
\tau(\alpha \tilde{u})=S_{\alpha} \tau(\tilde{u})
$$

in other words, we consider $\tau$ as a single valued holomorphic function on $\tilde{\Delta}^{\prime}$. Using (8), we define

$$
f_{\alpha}(\tilde{u})=\left(c_{\alpha} \tau(\tilde{u})+d_{\alpha}\right)^{-1}
$$


Next, we define the product $\tilde{\Delta}^{\prime} \times \mathbf{C}$ and, for each $\left(\alpha, n_{1}, n_{2}\right)$, with $\alpha \in \Pi_{1}\left(\Delta^{\prime}\right)$, and $n_{1}, n_{2}$ integers, the automorphism

$$
g\left(\alpha, n_{1}, n_{2}\right):(\tilde{u}, \lambda) \rightarrow\left(\alpha \tilde{u}, f_{\alpha}(\tilde{u})\left(\lambda+n_{1} \tau(\tilde{u})+n_{2}\right)\right)
$$

Denoting by $\mathcal{G}$ the group of automorphisms (11), we define the quotient space

$$
B^{\prime} \equiv\left(\tilde{\Delta}^{\prime} \times \mathcal{C}\right) / \mathcal{G}
$$

This is a non singular surface, since $g$, as defined by (11), has no fixed points in $\tilde{\Delta}^{\prime}$. From (11) and (12), it is clear that $B^{\prime}$ is an elliptic fibration on $\Delta^{\prime}$, with fiber elliptic curves of elliptic modulus $\tau(u)$. Thus, by the previous construction, we have defined the elliptic fibration away from the singular points $a_{\rho}$.

Let us denote $E_{\rho}$ a local neighbourhood of the point $a_{\rho}$, with local coordinate $t$, and such that $t\left(a_{\rho}\right)=0$. Let $S_{\rho}$ be the monodromy associated with a small circle around $a_{\rho}$. By $\mathcal{U}_{\rho}$ we will denote the universal covering of $E_{\rho}^{\prime}=E_{\rho}-a_{\rho}$, with coordinate $\rho$ defined by

$$
\rho=\frac{1}{2 \pi i} \log t
$$

The analog of (9) will be

$$
\tau(\rho+1)=S_{\rho} \tau(\rho)
$$

If we go around the points $a_{\rho}, k$ times, we should act with $S_{\rho}^{k}$; hence, we parametrize each path by the winding number $k$. The group of automorphisms (11), reduced to small closed paths around $a_{\rho}$, becomes

$$
g\left(k, n_{1}, n_{2}\right)(\rho, \lambda)=\left(\rho+k, f_{k}(\rho)\left[\lambda+n_{1} \tau(\rho)+n_{2}\right]\right) .
$$

Denoting by $\mathcal{G}_{\rho}$ the group $(15)$, we define the elliptic fibration around $a_{\rho}$ as

$$
\left(\mathcal{U}_{\rho} \times \mathbf{C}\right) / \mathcal{G}_{\rho}
$$

Next, we will extend the elliptic fibration to the singular point $a_{\rho}$. We can consider two different cases, depending on the finite or infinite order of $S_{\rho}$. 


\subsection{Singularities of Type $\hat{D}_{4}: \mathrm{Z}_{2}$ Orbifolds.}

Let us assume $S_{\rho}$ is of finite order,

$$
\left(S_{\rho}\right)^{m}=\mathbf{1}_{d}
$$

In this case, we can extend (16) to the singular points, simply defining a new variable $\sigma$ as

$$
\sigma^{m}=t
$$

Let us denote $D$ a local neighbourhood in the $\sigma$-plane of the point $\sigma=0$, and define the group $G_{D}$ of automorphisms

$$
g\left(n_{1}, n_{2}\right):(\sigma, \lambda)=\left(\sigma, \lambda+n_{1} \tau(\sigma)+n_{2}\right)
$$

and the space

$$
F=(D \times \mathbf{C}) / G_{D}
$$

Obviously, $F$ defines an elliptic fibration over $D$, with fiber $F_{\sigma}$ at each point $\sigma \in D$, an elliptic curve of modulus $\tau(\sigma)$. From (17) and (10), it follows that

$$
f_{k}(\sigma)=1
$$

with $k=O(m)$. Thus, we can define a normal subgroup $\mathcal{N}$ of $\mathcal{G}_{\rho}$ as the set of transformations (15):

$$
g\left(k, n_{1}, n_{2}\right):(\rho, \lambda) \rightarrow\left(\rho+k, \lambda+n_{1} \tau(\rho)+n_{2}\right)
$$

Comparing now (19) and (22), we get

$$
\left(\mathcal{U}_{\rho} \times \mathbf{C}\right) / \mathcal{N}=\left(D^{\prime} \times \mathbf{C}\right) / G_{D} \equiv F-F_{0}
$$

Using (22) and (15) we get

$$
\mathcal{C}=\mathcal{G} / \mathcal{N}
$$

with $\mathcal{C}$ the cyclic group of order $m$, defined by

$$
g_{k}:(\sigma, \lambda) \rightarrow\left(e^{2 \pi i k / m} \sigma, f_{k}(\sigma) \lambda\right)
$$


From (24) and (23), we get the desired extension to $a_{\rho}$, namely

$$
F / \mathcal{C}=\left(\mathcal{U}_{\rho} \times \mathbf{C}\right) / \mathcal{G}_{\rho} \cup F_{0} / \mathcal{C}
$$

Thus, the elliptic fibration extended to $a_{\rho}$, in case $S_{\rho}$ is of finite order, is defined by $F / \mathcal{C}$. Now, $F / \mathcal{C}$ can have singular points that we can regularize. The simplest example corresponds to

$$
S_{\rho}=\left(\begin{array}{cc}
-1 & 0 \\
0 & -1
\end{array}\right)
$$

i. e., a parity transformation. In this case, the order is $m=2$, and we define $\sigma$ by $\sigma^{2} \equiv t$. The cyclic group (25) in this case simply becomes

$$
(\sigma, \lambda) \rightarrow(-\sigma,-\lambda)
$$

since from (27) and (10) we get $f_{1}=-1$. At the point $\sigma=0$ we have four fixed points, the standard $\mathbf{Z}_{2}$ orbifold points,

$$
\left(0, \frac{a}{2} \tau(0)+\frac{b}{2}\right),
$$

with $a, b=0,1$. The resolution of these four singular points will produce four irreducible components, $\Theta^{1}, \ldots, \Theta^{4}$. In addition, we have the irreducible component $\Theta_{0}$, defined by the curve itself at $\sigma=0$. Using the relation $\sigma^{2}=t$, we get the $\hat{D}_{4}$ cycle,

$$
\mathcal{C}=2 \Theta_{0}+\Theta^{1}+\Theta^{2}+\Theta^{3}+\Theta^{4}
$$

with $\left(\Theta_{0}, \Theta^{1}\right)=\left(\Theta_{0}, \Theta^{2}\right)=\left(\Theta_{0}, \Theta^{3}\right)=\left(\Theta_{0}, \Theta^{4}\right)=1$. In general, the four external points of $D$-diagrams can be associated with the four $\mathbf{Z}_{2}$ orbifold points of the torus.

\subsection{Singularities of Type $\hat{A}_{n-1}$.}

We will now consider the case

$$
S_{\rho}=\left(\begin{array}{cc}
1 & n \\
0 & 1
\end{array}\right),
$$

which is of infinite order. A local model for this monodromy can be defined by

$$
\tau(t)=\frac{1}{2 \pi i} n \log t
$$


Using the variable $\rho$ defined in (13), we get, for the group $\mathcal{G}_{\rho}$ of automorphisms,

$$
g\left(k, n_{1}, n_{2}\right):(\rho, \lambda) \rightarrow\left(\rho+k, \lambda+n_{1} n \rho+n_{2}\right),
$$

and the local model for the elliptic fibration, out of the singular point,

$$
\left(\mathcal{U}_{\rho} \times \mathbf{C}\right) / \mathcal{G}_{\rho}
$$

i. e., fibers of the type of elliptic curves, with elliptic modulus $n \rho$. A simple way to think about these elliptic curves is in terms of cyclic unramified coverings [26]. Let us recall that a cyclic unramified covering, $\Pi: \hat{C} \rightarrow C$, of order $n$, of a curve $C$ of genus $g$, is a curve $\hat{C}$ of genus

$$
\hat{g}=n g+1-n
$$

Thus, for $g=1$, we get $\hat{g}=1$, for arbitrary $n$. Denoting by $\tau$ the elliptic modulus of $C$, in case $g=1$, the elliptic modulus of $\hat{C}$ is given by

$$
\hat{\tau}=n \tau
$$

Moreover, the generators $\hat{\alpha}$ and $\hat{\beta}$ of $H_{1}(\hat{C} ; \mathbf{Z})$ are given in terms of the homology basis $\alpha, \beta$ of $C$ as

$$
\begin{aligned}
& \Pi \hat{\alpha}=\alpha, \\
& \Pi \hat{\beta}=n \beta
\end{aligned}
$$

with $\Pi$ the projection $\Pi: \hat{C} \rightarrow C$. From (36) and (33), we can interpret the elliptic fibration (34) as one with elliptic fibers given by $n$-cyclic unramified coverings of a curve $C$ with elliptic modulus $\rho$ or, equivalently, $\frac{1}{2 \pi i} \log t$. There exits a simple way to define a family of elliptic curves, with elliptic modulus given by $\frac{1}{2 \pi i} \log t$, which is the plumbing fixture construction. Let $D_{0}$ be the unit disc around $t=0$, and let $C_{0}$ be the Riemann sphere. Define two local coordinates, $z_{a}: \mathcal{U}_{a} \rightarrow D_{0}, z_{b}: \mathcal{U}_{b} \rightarrow D_{0}$, in disjoint neigbourhoods $\mathcal{U}_{a}$, $\mathcal{U}_{b}$, of two points $P_{a}$ and $P_{b}$ of $\mathcal{C}_{0}$. Let us then define

$$
\begin{gathered}
W=\left\{(p, t) \mid t \in D_{0}, p \in C_{0}-\mathcal{U}_{a}-\mathcal{U}_{b} \text {, or } p \in \mathcal{U}_{a} \text {, with }\left|z_{a}(p)\right|>|t|\right. \text {, or } \\
\left.\qquad p \in \mathcal{U}_{b} \text {, with }\left|z_{b}(p)\right|>|t|\right\},
\end{gathered}
$$


and let $S$ be the surface

$$
S=\left\{x y=t ;(x, y, t) \in D_{0} \times O_{0} \times D_{0}\right\}
$$

We define the family of curves through the following identifications

$$
\begin{aligned}
\left(p_{a}, t\right) \in W \cap \mathcal{U}_{a} \times D_{0} & \simeq\left(z_{a}\left(p_{a}\right), \frac{t}{z_{a}\left(p_{a}\right)}, t\right) \in S \\
\left(p_{b}, t\right) \in W \cap \mathcal{U}_{b} \times D_{0} & \simeq\left(\frac{t}{z_{b}\left(p_{b}\right)}, z_{b}\left(p_{b}\right), t\right) \in S .
\end{aligned}
$$

For each $t$ we get a genus one curve, and at $t=0$ we get a nodal curve by pinching the non zero homology cycles. The pinching region is characterized by

$$
x y=t
$$

which defines a singularity of type $A_{0}$. The elliptic modulus of the curves is given by

$$
\tau(t)=\frac{1}{2 \pi i} \log t+C_{1} t+C_{2}
$$

for some constants $C_{1}$ and $C_{2}$. We can use an appropiate choice of coordinate $t$, such that $C_{1}=C_{2}=0$. The singularity at $t=0$ is a singularity of type $\hat{A}_{0}$, in Kodaira's classification, corresponding to

$$
S_{\rho}=\left(\begin{array}{ll}
1 & 1 \\
0 & 1
\end{array}\right)
$$

Using now (36) and (42) we get, for the cyclic covering of order $n$, the result (32), and the group (33). The pinching region of the cyclic unramified covering is given by

$$
x y=t^{n},
$$

instead of (41), i. e., for the surface defining the $A_{n-1}$ singularity, $\mathbf{C}^{2} / \mathbf{Z}_{n}$. Now, we can proceed to the resolution of the singularity at $t=0$. The resolution of the singularity (44) requires $n-1$ exceptional divisors, $\Theta_{1}, \ldots, \Theta_{n-1}$. In addition, we have the rational curve $\Theta_{0}$, defined by the complement of the node. Thus, we get, at $t=0$,

$$
\mathcal{C}=\Theta_{0}+\cdots+\Theta_{n-1},
$$


with $\left(\Theta_{0}, \Theta_{1}\right)=\left(\Theta_{0}, \Theta_{n-1}\right)=1$, and $\left(\Theta_{i}, \Theta_{i+1}\right)=1$, which is the $\hat{A}_{n-1}$ Dynkin diagram. The group of covering transformations of the $n^{\text {th }}$ order cyclic unramified covering is $\mathbf{Z}_{n}$, and the action over the components 45 is given by

$$
\begin{aligned}
\Theta_{i} & \rightarrow \Theta_{i+1}, \\
\Theta_{n-1} & \rightarrow \Theta_{0} .
\end{aligned}
$$

\subsection{Singularities of Type $\hat{D}_{n+4}$.}

This case is a combination of the two previous examples. Through the same reasoning as above, the group $\mathcal{G}_{\rho}$ is given, for

$$
S_{\rho}=\left(\begin{array}{cc}
-1 & -n \\
0 & -1
\end{array}\right) .
$$

by

$$
g\left(k, n_{1}, n_{2}\right):(\rho, \lambda) \rightarrow\left(k+\rho,(-1)^{k}\left(\lambda+n_{1} n \rho+n_{2}\right)\right) .
$$

Using a new variable $\sigma^{2}=t$, what we get is a set of irreducible components $\Theta_{0}, \ldots \Theta_{2 n}$, with the identifications $\Theta_{i} \rightarrow \Theta_{2 n-i}$. In addition, we get the four fixed $\mathbf{Z}_{2}$ orbifold points described above. The singular fiber is then given by

$$
\mathcal{C}=2 \Theta_{0}+\cdots+2 \Theta_{n}+\Theta^{1}+\Theta^{2}+\Theta^{3}+\Theta^{4}
$$

with the intersections of the $\hat{D}_{n+4}$ affine diagram. It is easy to see that in this case we also get

$$
(\mathcal{C})^{2}=0
$$

Defing the genus of the singular fiber by $C^{2}=2 g-2$, we conclude that $g=1$, for

all singularities of Kodaira type. Notice that for rational singularities, characterized by non affine Dynkin diagrams of ADE type [27, we get self intersection $\mathcal{C}^{2}=-2$, which corresponds to genus equal zero.

\section{Decompactification and Affinization.}

The general framework in which we are working in order to get four dimensional $N=1$ gauge theories is that of M-theory compactifications on elliptically fibered Calabi-Yau 
fourfolds, in the limit $\operatorname{Vol}(E)=0$, with $E$ the elliptic fiber. As described above, we can interpolate between $N=2$ supersymmetry in three dimensions, and $N=1$ in four dimensions, by changing the radius $R$ through

$$
\operatorname{Vol}(E)=\frac{1}{R}
$$

The three dimensional limit then corresponds to $\operatorname{Vol}(E) \rightarrow \infty$, and the four dimensional to $\operatorname{Vol}(E) \rightarrow 0$. Now, we will work locally around a singular fiber of Kodaira $\hat{A} \hat{D} \hat{E}$ type. As we know, for the Calabi-Yau fourfold $X$,

$$
E \rightarrow X \stackrel{\Pi}{\rightarrow} B
$$

the locus $C$ in $B$, where the fiber is singular, is of codimension one in $B$, i. e., of real dimension four. Let us now see what happens to the singular fiber in the three dimensional limit. In this case, we have $\operatorname{Vol}(E)=\infty$. A possible way to represent this phenomenon is by simply extracting the point at infinity. In the case of $\hat{A}_{n-1}$ singularities, as described in previous subsection, taking out the point at infinity corresponds to decompactifying the irreducible component $\Theta_{0}$, that was associated with the curve itself. As was clear in this case, we then pass from the affine diagram, $\hat{A}_{n-1}$, to the non affine, $A_{n-1}$. More generally, as the elliptic fibration we are considering possesses a global section, we can select the irreducible component we are going to decompactify as the one intersecting with the basis of the elliptic fibration. When we decompactify, in the $\operatorname{Vol}(E)=0$ limit, what we are doing, at the level of the fiber, is precisely compactifying the extra irreducible component, which leads to the affine Dynkin diagram.

\section{M-Theory Instantons and Holomorphic Euler Char- acteristic.}

Using the results of reference [1] a vertical instanton [1] in a Calabi-Yau fourfold, of the type (52), will be defined by a divisor $D$ of $X$, such that $\Pi(D)$ is of codimension one in $B$, and with holomorphic Euler characteristic

$$
\chi\left(D, \mathcal{O}_{D}\right)=1
$$


It is in case (53) that we have two fermionic zero modes [1] , and we can define a superpotential contribution associated to $D$. For $N$, the normal bundle to $D$ in $X$, which is locally a complex line bundle on $D$, we define the $U(1)$ transformation

$$
t \rightarrow e^{i \alpha} t
$$

with $t$ a coordinate of the fiber of $N$. The two fermionic zero modes have $U(1)$ charge equal one half. Associated to the divisor $D$, we can define a scalar field $\phi_{D}$ that, together with $\mathrm{Vol}(D)$ defines the imaginary and real parts of a chiral superfield. Under $U(1)$ rotations (54), $\phi_{D}$ transforms as

$$
\phi_{D} \rightarrow \phi_{D}+\chi(D) \alpha
$$

In three dimensions, this is precisely the transformation of the dual photon field as Goldstone boson [22. However, transformation (55) has perfect sense, for vertical instantons, in the four dimensional decompactification limit.

Let us now consider an elliptically fibered Calabi-Yau fourfold, with singular fiber of $\hat{A}_{n-1}$ type, over a locus $C$ of codimension one in $B$. We will assume that the singular fiber is constant over $C$. Moreover, in the geometrical engineering spirit, we will impose

$$
h_{1,0}(C)=h_{2,0}(C)=0
$$

and, thus, $\Pi_{1}(C)=0$. This prevents us from having non trivial transformations on the fiber by going, on $C$, around closed loops, since all closed loops are contractible. In addition, we will assume, based on (56), that $C$ is an Enriques surface. After impossing these assumptions, we will consider divisors $D_{i}$, with $i=0, \ldots, n-1$, defined by the fibering over $C$, in a trivial way, of the irreducible components $\Theta_{i}$ of the $\hat{A}_{n-1}$ singularity [7]. Ussing the Todd representation of the holomorphic Euler class [23,

$$
\chi(D)=\frac{1}{24} \int_{D_{i}} c_{1}\left(\Theta_{i}\right) c_{2}(C)
$$

we get, for $C$ an Enriques surface,

$$
\chi\left(D_{i}\right)=1 .
$$


Interpreting now the $t$ variable (54) on the fiber of the normal bundle $N$ of $D$ in $X$ as the $t$ variable used in our previous description of Kodaira singularities of type $\hat{A}_{n-1}$, we can derive the transformation law, under the $\mathbf{Z}_{n}$ subgroup of $U(1)$, of the scalar fields $\phi_{D_{i}}$ associated to these divisors. Namely, from (46) we get

$$
\mathbf{Z}_{n}: \phi_{D_{i}} \rightarrow \phi_{D_{i+1}}
$$

with the $\mathbf{Z}_{n}$ transformation being defined by

$$
t \rightarrow e^{2 \pi i / n} t
$$

Using now (55), we get

$$
\mathbf{Z}_{n}: \phi_{D_{i}} \rightarrow \phi_{D_{i}}+\frac{2 \pi}{n}
$$

Combining (59) and (61) we get, modulo $2 \pi$,

$$
\phi_{D_{j}}=\frac{2 \pi j}{n}+c
$$

with $j=0, \ldots, n-1$, and $c$ a constant independent of $j$.

Let us now consider the divisor $\mathcal{D}$ obtained by fibering over $C$ the singular fiber $\mathcal{C}=\sum_{j=0}^{n-1} \Theta_{j}$, defined in (45). In this case we need to be careful in order to compute (57). If we naively consider the topological sum of components $\Theta_{j}$ in (57), we will get the wrong result $\chi(\mathcal{D})=n$. This result would be correct topologically, but not for the holomorphic Euler characteristic we are interested in. In fact, what we should write in (57) for $\int c_{1}\left(\sum_{j=0}^{n-1} \Theta_{j}\right)$ is $2\left(1-g\left(\sum_{j=0}^{n-1} \Theta_{j}\right)\right)$, with $g$ the genus of the cycle (45), as defined by $\mathcal{C}^{2}=2 g-2$, with $\mathcal{C}^{2}$ the self intrsection of the cycle 45 which, as for any other Kodaira singularity, is zero. Thus, we get $g=1$, and

$$
\chi(\mathcal{D})=0
$$

We can try to intepret the result (63) in terms of the fermionic zero modes of each component $\Theta_{i}$, and the topology of the cycle. In fact, associated to each divisor $D_{i}$ we have, as a consequence of (58), two fermionic zero modes. In the case of the $\hat{A}_{n-1}$ singularity, we can soak up all zero modes inside the graph, as shown in Figure 1,

where from each node, representing one $\Theta_{i}$, we have two fermionic zero mode lines. The soaking up of fermionic zero modes represented in the figure is an heuristic interpretation of the result $(63)$. 


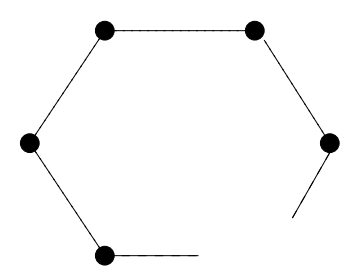

fermionic zero mode

Figure 1: Soaking up of zero modes for $\hat{A}_{n-1}$.

\section{$5 \quad \theta$-Parameter and Gaugino Condensates.}

We will, in this section, only consider singularities of $\hat{A}_{n-1}$ and $\hat{D}_{n+4}$ type. In both cases, and for each irreducible component $\Theta_{i}$, we get a divisor $D_{i}$, with $\chi\left(D_{i}\right)=1$. Associated to this divisor, we can get a superpotential term of the order [1]

$$
\int d^{2} \theta e^{\left.-\left(V\left(D_{i}\right)\right)+i \phi_{D_{i}}\right)}
$$

where $V\left(D_{i}\right)$ means the volume of the divisor $D_{i}$. As explained above, we are using vertical instanton divisors $D_{i}$, defined by a trivial fibering of $\Theta_{i}$ over the singular locus $C \subset B$, satisfying conditions (56). In order to get the four dimensional $N=1$ limit, we will take the limit $\operatorname{Vol}(E)=\frac{1}{R} \rightarrow 0$. Since the singular fibers are, topologically, the union of irreducible components (see (45) and (49)), we can write

$$
\operatorname{Vol}\left(\Theta_{i}\right)=\frac{1}{R \operatorname{Cox}}
$$

with Cox the Coxeter number of the corresponding singularity, which equals the total number of irreducible components. Therefore, we will define $\operatorname{Vol}\left(D_{i}\right)$ as

$$
\operatorname{Vol}\left(D_{i}\right)=\lim _{R \rightarrow \infty} \operatorname{Vol}(C) \frac{1}{R \operatorname{Cox}}
$$

If we first consider the $N=2$ supersymmetric three dimensional theory obtained by compactifying M-theory on the Calabi-Yau fourfold $X$, i. e., in the limit $R \rightarrow 0$, we know that only the divisor $\Theta_{0}$, for the $\hat{A}_{n-1}$ case, is decompactified, passing from the affine diagram describing an elliptic singularity to the non affine diagram describing a 
rational, Artin like, singularity [27]. In that case, the volumes of the $\Theta_{i}$ components, for $i \neq 0$, are free parameters, corresponding to the Coulomb branch of the $N=2$ three dimensional theory. In the three dimensional theory, the factor $\operatorname{Vol}(C)$ corresponds to the bare coupling constant in three dimensions,

$$
\operatorname{Vol}(C)=\frac{1}{g_{3}^{2}}
$$

and $\operatorname{Vol}\left(D_{i}\right)=\frac{1}{g_{3}^{2}} \chi_{i}$, for $i \neq 0$, with $\chi_{i}$ the three dimensional Coulomb branch coordinates. In the four dimensional case, we must use (66), that becomes

$$
\operatorname{Vol}\left(D_{i}\right)=\lim _{R \rightarrow \infty} \frac{1}{g_{3}^{2}} \frac{1}{R \operatorname{Cox}}=\frac{1}{g_{4}^{2} \operatorname{Cox}} \text {. }
$$

Let us now concentrate on the $\hat{A}_{n-1}$ case, where Cox $=n$. Using (64) we get the following superpotential for each divisor $D_{j}$,

$$
\exp -\left(\frac{1}{g_{4}^{2} n}+i\left(\frac{2 \pi j}{n}+c\right)\right) .
$$

Let us now fix the constant $c$ in $(\sqrt[69]{6})$. In order to do that, we will use the transformation rules (55). From the four dimensional point of view, these are the transformation rules with respect to the $U(1)_{R}$ symmetry. From (55) we get, that under $t \rightarrow e^{i \alpha} t$,

$$
\sum_{i=0}^{\mathrm{n}-1} \phi_{D_{i}} \rightarrow \sum_{i=0}^{\mathrm{n}-1} \phi_{D_{i}}+n \alpha .
$$

This is precisely the transformation rule under $U(1)_{R}$ of the $N=1 \theta$-parameter,

$$
\theta \rightarrow \theta+n \alpha
$$

In fact, (70) is a direct consequence of the $U(1)$ axial anomaly equation: if we define $\theta$ as

$$
\frac{\theta}{32 \pi^{2}} F \tilde{F}
$$

the anomaly for $S U(n)$ is given by

$$
\partial_{\mu} j_{5}^{\mu}=\frac{n}{16 \pi^{2}} F \tilde{F}
$$


The factor 2, differing (72) from (73), reflects the fact that we are assigning $U(1)_{R}$ charge $\frac{1}{2}$ to the fermionic zero modes. Identifying the $\theta$-parameter with the topological sum $\sum_{i=0}^{n-1} \phi_{D_{i}}$ we get that the constant $c$ in $(69)$ is simply

$$
\mathrm{c}=\frac{\theta}{n}
$$

so that we then finally obtain the superpotential

$$
\exp -\left(\frac{1}{g_{4}^{2} n}+i\left(\frac{2 \pi j}{n}+\frac{\theta}{n}\right)\right) \simeq \Lambda^{3} e^{2 \pi i j / n} e^{i \theta / n}
$$

with $j=0, \ldots, n-1$, which is the correct value for the gaugino condensate.

Let us now try to extend the previous argument to the $\hat{D}_{n+4}$ type of singularities. Defining again the four dimensional $\theta$-parameter as the topological sum of $\phi_{D_{i}}$ for the whole set of irreducible components we get, for the cycle (49), the transformation rule

$$
\theta \rightarrow \theta+\operatorname{Cox} \cdot \alpha
$$

where now the Coxeter for $\hat{D}_{n+4}$ is $2 n+6$. Interpreting $\hat{D}_{n+4}$ as $O(N)$ gauge groups, with $N=2 n+8$, we get $\operatorname{Cox}\left(\hat{D}_{n+4}\right)=N-2$. Since $\theta$ is defined modulo $2 \pi$ we get that for $\hat{D}_{n+4}$ singularities the value of $\phi_{D_{i}}$, for any irreducible component, is

$$
\frac{2 \pi k}{N-2}+\frac{\theta}{N-2}
$$

with $k=1, \ldots, N-2$. However, now we do not know how to associate a value of $k$ to each irreducible component $\Theta_{i}$ of the $\hat{D}_{n+4}$ diagram. Using (77), we get a set of $N-2$ different values for the gaugino condensate for $O(N)$ groups:

$$
\exp \left(-\frac{1}{g_{4}^{2}(N-2)}+i\left(\frac{2 \pi k}{N-2}+\frac{\theta}{N-2}\right)\right)
$$

with $k=1, \ldots, N-2$. However, we still do not know how to associate to each $\Theta_{i}$ a particular value of $k$. A possibility will be associating consecutive values of $k$ to components with non vanishing intersection; however, the topology of diagrams of type $D$ prevents us from doing that globally. Notice that the problem we have is the same sort of puzzle we find for $O(N)$ gauge groups, concerning the number of values for $\langle\lambda \lambda\rangle$, 
and the value of the Witten index, which in diagramatic terms is simply the number of nodes of the diagram. In order to unravel this puzzle, let us consider more closely the way fermionic zero modes are soaked up on a $\hat{D}_{n+4}$ diagram. We will use the cycle (49); for the components $\Theta^{1}$ to $\Theta^{4}$, associated to the $\mathbf{Z}_{2}$ orbifold points, we get divisors with $\chi=1$. Now, for the components $2 \Theta_{0}, \ldots, 2 \Theta_{n}$ we get, from the Todd representation of the holomorphic Euler characteristic,

$$
\chi=4
$$

The reason for this is that the cycle $2 \Theta$, with $\Theta^{2}=-2$, has self intersection -8 . Of course, (79) refers to the holomorphic Euler characteristic of the divisor obtained when fibering over $C$ any of the cycles $2 \Theta_{i}$, with $i=0, \ldots, n$. Equation (79) implies 8 fermionic zero modes, with the topology of the soaking up of zero modes of the $\hat{D}_{n+4}$ diagram, as represented in Figure 2.

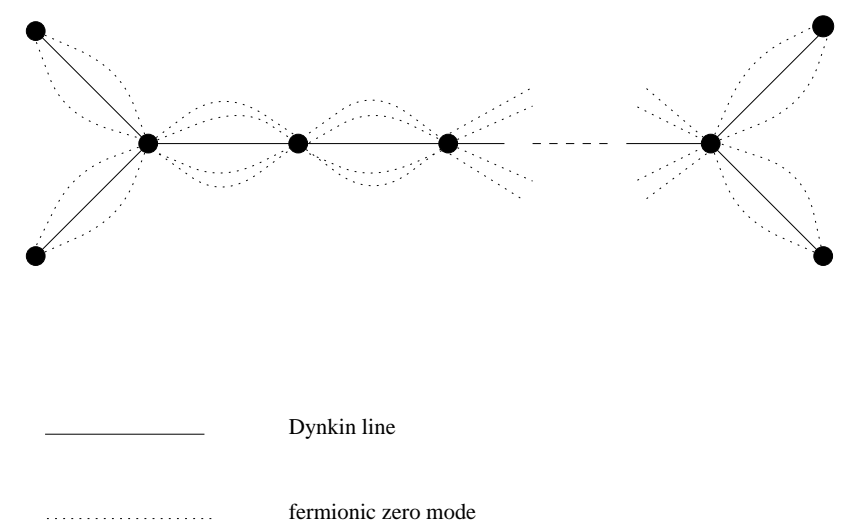

Figure 2: Soaking up of zero modes for $\hat{D}_{n+4}$.

Notice that the contribution to $\chi$ of $2 \Theta$ is different form that of $\left(\Theta_{1}+\Theta_{2}\right)$, with $\left(\Theta_{1} \cdot \Theta_{2}\right)=0$; namely, for the first case $\chi=4$, and $\chi=2$ for the second. For the $\hat{D}_{n+4}$ diagram, we can define: $i$ ) The Witten index $\operatorname{tr}(-1)^{F}$, as the number of nodes, i. e., $5+n ; i i)$ The Coxeter number, which is the number of irreducible components, i. e., $2 n+6$ and $i i i)$ The number of intersections as represented by the dashed lines in Figure 4 , i. e., $8+4 n$. From the point of view of the Cartan algebra, used to define the vacuum configurations in [9], we can only feel the number of nodes. The $\theta$-parameter is able to feel 
the Coxeter number; however, we now find a new structure related to the intersections of the graph. In the Witten index case, the nodes corresponding to cycles $2 \Theta_{i}$, with $i=0, \ldots, n$ contribute with one, in the number of $\langle\lambda \lambda\rangle$ values with two, and in the number of intersections with four. This value four calls for an orientifold interpretation of these nodes. The topological definition of the $\theta$-parameter implicitely implies the split of this orientifold into two cycles, a phenomena recalling the F-theory description [29] of the Seiberg-Witten splitting [28]. Assuming this splitting of the orientifold, the only possible topology for the soaking up of zero modes is the one represented in Figure 3, where the "splitted orientifold" inside the box is associated to four zero modes, corresponding to $\chi=2$ for a cycle $\Theta_{1}+\Theta_{2}$, with $\Theta_{1} \cdot \Theta_{2}=0$.

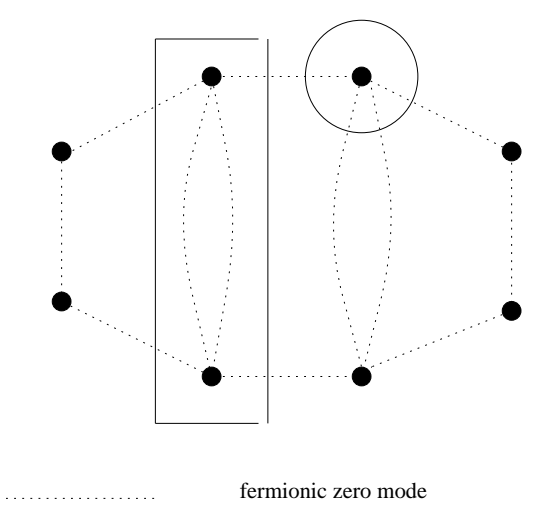

Figure 3: Orientifold splitting.

On the other hand, each node surrounded by a circle in Figure 5 represents itself the disconnected sum of two non singular rational curves; thus, we represent each "orientifold" mode by four rational curves, with the intersections depicted inside the box of Figure 5 . When we forget about internal lines in Figure 5, we get the cyclic $\mathbf{Z}_{2 n+6} \equiv \mathbf{Z}_{N-2}$ structure of equation (78). It is clear that much more is necessary in order to reach a complete description of the $O(N)$ vacuum structure.

\section{Domain Walls and Intersections.}

The discussion in the previous section already raises the problem known as $\theta$-puzzle. In fact, and discussing again only the $S U(n)$ case, the transformation law (71) together with 
the very definition fo the $\theta$-angle as the topological sum $\sum_{i=0}^{n-1} \phi_{D_{i}}$ would imply that $\theta$ is the scalar field $\phi_{\mathcal{D}}$ of the 6 -cycle associated to the $\hat{A}_{n-1}$ cycle, $\mathcal{C}=\sum_{i=0}^{n-1} \Theta_{i}$. On the basis of (55), this will be equivalent to saying that $\chi(\mathcal{D})=n$, instead of zero. This is, in mathematical terms, the $\theta$-puzzle. The mathematical solution comes from the fact that $\chi(\mathcal{D})=0$. In this section we will relate this result, on the value of the holomorphic Euler chareacteristic, to the appearance of domain walls 110, 11. To start with, let us consider a cycle $\mathcal{C}=\Theta_{1}+\Theta_{2}$, with $\left(\Theta_{1} \Theta_{2}\right)=1$. The self intersection can be expressed as

$$
(\mathcal{C} . \mathcal{C})=-2-2+2
$$

where the -2 contributions come from $\Theta_{1}^{2}$ and $\Theta_{2}^{2}$, and the +2 comes from the intersection between $\Theta_{1}$ and $\Theta_{2}$. As usual, we can consider $\mathcal{C}$ trivially fibered on an Enriques surface. The holomorphic Euler chracteristic of the corresponding six cycle can be written as

$$
\chi=\frac{1}{2}\left(-\mathcal{C}^{2}\right) .
$$

Using now the decomposition (80) we get two contributions of one, coming from the components $\Theta_{1}$ and $\Theta_{2}$, considered independently, and a contribution of -1 from the intersection term +2 in (80). In this sense, the intersection term can be associated to two fermionic zero modes, and net change of chiral charge oposite to that of the $\Theta_{i}$ components. When we do this for the cycle $\mathcal{C}$ of $\hat{A}_{n-1}$ singularities, we get that each intersection is soaking up two zero modes, leading to the result that $\chi(\mathcal{C})=0$. A graphical way to represent equation (80) is presented in Figure 4.

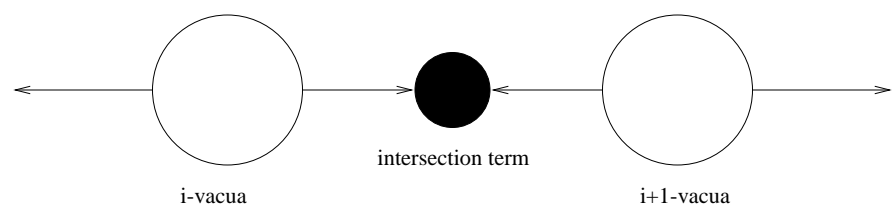

Figure 4: Intersection term.

Now, we will wonder about the physical interpretation of the intersection terms leading to $\chi(\mathcal{C})=0$ for all Kodaira singularities. The simplest, and most natural answer, is certainly domain walls extending between different vacua, or values of $\langle\lambda \lambda\rangle$. 
From the point of view of zero mode counting, the "intersection term" behaves effectively as an anti-instanton with two fermionic zero modes. One of these fermionic zero modes, let us say $\psi_{j, j+1}$, is associated to the intersection of $\Theta_{j}$ with $\Theta_{j+1}$ and the other $\psi_{j+1, j}$ with the intersection of $\Theta_{j}$ and $\Theta_{j+1}$. Thus, extending naively the computation done for irreducible components, the contribution of the black box in Figure 4 should be of the order

$$
\Lambda^{3} e^{2 \pi i j / n}\left(1-e^{2 \pi i / n}\right) .
$$

In result (82), interpreted as the contribution of the intersection term, the most surprising fact is the appearance of $\Lambda^{3}$, since now we are geometrically considering simply a point; the factor $\Lambda^{3}$ in the computation of the gaugino condensate comes from the volume of the divisor. In the same way as we interpret M-theory instantons as fivebranes wrapped on the six-cycles used to define the instanton, we can think of the intersection terms as fivebranes wrapped on the cycle $C \times\left\{\left(\Theta_{i} \cdot \Theta_{i+1}\right)\right\}$, i. e., the product of the singular locus $C$ and the intersection point. The fivebrane wrapped on this cycle defines, in four dimensions, a domain wall, let us say interwining between the vacua $i$, at $x_{3}=+\infty$, and the vacua $i+1$, at $x_{3}=-\infty$, where the coordinate $x_{3}$ is identified with the unwrapped direction. It is in this sense that we should use (82) to define the energy density, or tension, of the domain wall. In the four dimensional limit, $\operatorname{Vol}(E)$ goes zero as $\frac{1}{R}$; moreover, the local engineering approach works in the limit where the volume of the singular locus $C$ is very large, so that we can very likely assume that intersection terms behave like (82), with $\Lambda^{3}$, but only in the four dimensional limit. Cyclicity of the $\hat{A}_{n-1}$ diagram allows us to pass from the $j$ to the $j+1$ vacua in two different ways: $n-1$ steps, or a single one. The sum of both contributions should define the physical domain wall; thus the energy density will behave as

$$
n \Lambda^{3}\left|e^{2 \pi i j / n}\left(1-e^{2 \pi i / n}\right)\right|
$$

The extension of the previous argument to the case of $O(N)$ groups is certainly more involved, due to the topology of the $\hat{D}$ diagram, and the presence of orientifolds. It would certainly be interesting studying the interplay between orientifolds and domain walls in this case. 
Finally, we will say some words on the QCD string. In reference [14, the geometry of QCD strings is intimately related to the topological fact that

$$
H_{1}(Y / Z ; \mathbf{Z})=\mathbf{Z}_{n}
$$

where $\Sigma$ is a rational curve associated to the configuration of fourbranes, and $Y=S^{1} \times \mathbf{R}^{5}$ is the ambient space where $\Sigma$ is embedded (see [14] for details). The QCD string is then associated to a partially wrapped membrane on a non trivial element of $H_{1}(Y / \Sigma ; \mathbf{Z})$. Recall that $H_{1}(Y / \Sigma ; \mathbf{Z})$ is defined by one-cycles in $Y$, with boundary on $\Sigma$. The previous discussion was done for $S U(N)$ gauge groups. Using our model of $\hat{A}_{n-1}$ singularities, described in section 2, the analog in our framework of (84) is equation (37). Then we can, in the same spirit as in reference [14], associate the QCD string to paths going from $p_{k}$ to $p_{k+1}$, where $p_{k}$ are the intersection points,

$$
\Theta_{k} \cdot \Theta_{k-1}=p_{k}
$$

Geometrically, it is clear that the tension of this QCD string is the square root of the domain wall tension. By construction, the QCD string we are suggesting here ends on domain walls, i. e., on intersection points.

To end up, let us include some comments on the existence of extra vacua, as suggested in [13]. It is known that the strong coupling computation of $\langle\lambda \lambda\rangle$ does not coincide with the weak coupling computation; more precisely[16],

$$
<\lambda \lambda>_{\mathrm{sc}}<<\lambda \lambda>_{\mathrm{wc}}
$$

In the framework of M-theory instanton computations, the numerical factors will depend in particular on the moduli of complex structures of the Calabi-Yau fourfold. In the strong coupling regime we must consider structures preserving the elliptic fibration structure and the Picard lattice. In the weak coupling regime, where the compuatation is performed in the Higgs phase, the amount of allowed complex structures contributing to the value of $\langle\lambda \lambda\rangle$ is presumably larger. Obviously, the previous argument is only suggesting a possible way out of the puzzle (86).

Equally, at a very speculative level, the extra vacua, with no chiral symmetry breaking, could be associated to the cycle $\mathcal{D}$ defining the singular fiber, a cycle that we know leads 
to $\chi=0$, and therefore does not produce any gaugino condensate. Notice that any other cycle with $\chi \neq 0$ will lead, if clustering is used, to some non vanishing gaugino condensates, so that $\mathcal{D}$ with $\chi=0$ looks like a possible candidate to the extra vacua suggested in 13 . If this argument is correct this extra vacua will appears for any Kodaira singularity i. e. in any $\operatorname{ADE} N=1$ four dimensional gauge theory.

It is important to stress that the $\theta$-puzzle is not exclusive of $N=1$ gluodynamics. In the $N=0$ case the Witten-Veneziano formula [24, 25] for the $\eta^{\prime}$ mass also indicates a dependence of the vacuum energy on $\theta$ in terms of $\frac{\theta}{N}$, which means a set of entangled "vacuum" states. In our approach to $N=1$ the origin of this entanglement is due to the fact that $\chi=0$ for the singular cycle. In fact, $\chi(\mathcal{D})=0$ means that the set of divisors $D_{i}$, plus the intersections, i. e., the domain walls, are invariant under $U(1)$, as implied by equation (55). If we naively think of something similar in $N=0$ and we look for the

origin of vacuum entanglement in intersections we maybe should think in translating the topology of intersections into topological properties of abelian proyection gauges [30].

\section{Acknowledgments}

The author would like to thank U. Bruzzo , C. Reina and R. Hernandez for discussions and to U. Bruzzo for bringing my attention to reference [23]. This work is partially supported by European Community grant ERBFMRXCT960012, by grant AEN96-1655. 


\section{References}

[1] E. Witten, Nucl. Phys. B474 (1996), 343.

[2] S. Sethi, C. Vafa and E. Witten hep-th/9606122.

[3] R. Donagi, A. Grassi and E. Witten, hep-the/9607091.

[4] R. Gopakumar and S. Muki hep-th/9607057.

[5] K. Kodaira, Ann. Math. 77, 3 (1963), 563.

[6] S.Katz, A. Klemm and C. Vafa, hep-th/9609239.

[7] S. Katz and C. Vafa, hep-th/9611090.

[8] A. Smilga, hrp-th/9607007.

[9] E. Witten, Nucl. Phys. B202 (1982), 253.

[10] G.Dvali and M. Shifman hep.th/9612128.

[11] A. Kovner, M. Shifman and A. Smilga hep-th/9706089.

[12] A. Smilga and A. Veselov, hep-th/9710123 and hep-th/9706217.

[13] A. Kovner and M. Shifman, hep-th/9702174.

[14] E. Witten, hep-th/9706109.

[15] V. A. Novikov, M. A. Shifman, A. I. Vainshtein and V. I. Zakharov, Nucl. Phys. B229 (1983), 407.

[16] V. A. Novikov, M. A. Shifman, A. I. Vainshtein and V. I. Zakharov, Nucl. Phys. B260 (1985), 157.

[17] D. Amati, K. Konishi, Y. Meurice, G. C. Rossi and G. Veneziano, Phys. Rep. 162 (1988), 169. 
[18] E. Cohen and C. Gómez, Phys. Rev. Lett. 52 (1984), 237.

[19] G. 't Hooft, Commun. Math. Phys. 81 (1981), 267.

[20] M. A. Shifman and A. I. Vainshtein, Nucl. Phys. B296 (1988), 445.

[21] K. Intriligator and N. Seiberg, Nucl. Phys. Proc. Supl. 45 (1996), hep-th/9509066.

[22] I. Affleck, J. A. Harvey and E. Witten, Nucl. Phys. B206 (1982), 413.

[23] W. Fulton, "Intersection Theory", Springer-Verlag, 1980.

[24] E. Witten, Nucl. Phys. B156 (1979), 269.

[25] G. Veneziano, Nucl. Phys. B159 (1979), 213.

[26] J. Fay, Lect. Not. in Math. 352.

[27] M. Artin, Amer. J. Math. 88 (1966), 129.

[28] N. Seiberg and E. Witten, Nucl. Phys. B426 (1994), 19.

[29] A. Sen, Nucl. Phys. B475 (1996), 562; hep-th/9605150.

[30] G. 't Hooft, Nucl. Phys. B190 (1981), 455. 